\title{
The prototype dosimetry system to protect MPD electronic equipment at the new NICA collider
}

\author{
Marcin Bielewicz ${ }^{1,2, *}$, Arkadiusz Chlopik ${ }^{1}$, Aleksandr Bancer ${ }^{1}$, Adam Dudzinski ${ }^{1}$, Elzbieta Jaworska ${ }^{1}$, Jacek \\ Rzadkiewicz $^{1}$, Krystian Roslon ${ }^{2,3},{\text { Nikita } \text { Dunin }^{2} \text {, and Michalina Milewicz-Zalewska }}^{2,3}$ \\ ${ }^{1}$ National Centre for Nuclear Research, Swierk, Poland \\ ${ }^{2}$ Joint Institute of Nuclear Research, Dubna, Russia \\ ${ }^{3}$ Warsaw University of Technology, Warsaw, Poland
}

\begin{abstract}
The Multi-Purpose Detector (MPD) is a main detection system of the new collider located in Dubna, Russia (Nuclotron-based Ion Collider fAcility -NICA). During the work, the Slow Control electronic equipment which is located on the MPD surface and on the special platform near the MPD body, an accidental irradiation caused by the NICA's failure or its abnormal functioning may occur. Thus, there is a risk of destroying the electronics by a radiation exposure in the platform area, and in the consequence the emergency/fast switch off of the MPD sub-detectors might become impossible. We present the preliminary dosimetry system i.e. the method of prevention of such situation by the continuous monitoring on the Slow Control electronics on the platform. System will be alarming when the radiation levels threshold will be surpassed.
\end{abstract}

\section{Introduction}

The main detection system of the new collider NICA (Nuclotron-based Ion Collider fAcility)[1], is the MPD (Multi-Purpose Detector)[2]. The accelerators complex is designed at the Joint Institute for Nuclear Research in Dubna, Russia to study particle properties in the dense baryonic matter, and consist of: heavy and light ion sources with linear accelerators, 1-st accelerator ring BOOSTER, 2-nd accelerator ring NUCLOTRON, experimental stand BM@N, collider ring NICA and SPD, MPD detector sets. The MPD is located on a ground level in the concrete building. The work of MPD is controlled by special electronic equipment called Slow Control System[3]. The racks with electronic systems will be located on the MPD surface and on the special platform which is standing near the MPD (Fig. 1). The Dosimetry System to Protect MPD Electronic Equipment has two main roles to play. One is to monitor continuously the electronic devices placed inside the 19" RACKs which stand on the platform. In case of the unexpected rise of the radiation level after any NICA's failure or its abnormal functioning, the system should alarm the indicated persons by e-mail and/or mobile application. The second role is to monitor the air outside the crates in order to protect employees working on the platform. The radiation can destroy the Slow Control electronic elements and control over MPD would be lost. To prevent the MPD malfunctioning continuous monitoring of the level of radiation in the Slow Control room is needed.

*e-mail: marcin.bielewicz@ncbj.gov.pl
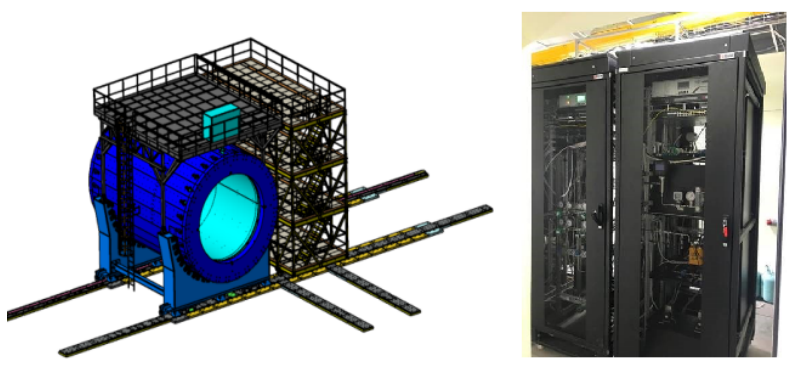

Figure 1. Left: MPD detectors and platform for MPD electronics and Slow Control System [2]. Right: RACK for Gas Supply System.

\section{System description}

The process or method of measuring the dosage of ionizing radiation by means of a dosimeter is called dosimetry. The dosimeter is an instrument for measuring and monitoring exposure to doses of radiation, such as $\mathrm{X}$-rays or gamma rays. The dosimetry system should continuously monitor the ionizing radiation level in the chamber taking into account two levels: the safe level for people working in that chamber and the level which can destroy the electronic devices. The results of the measurements need to be displayed on a computer screen instantly in the graphical manner (e.g. a chart or graph) and they should also be archived. When the measured doses exceed the threshold determined for electronics, a supervisory system should be automatically informed and proceed to switch off the devices successively with the specific procedure. It will allow us to avoid dangerous 
Table 1. Main information about detectors.

\begin{tabular}{|l|l|l|l|}
\hline & $\begin{array}{l}\text { Gamma- } \\
\text { Scout }\end{array}$ & EKO-C & EGM-104 \\
\hline Meas.Range & $\begin{array}{l}100 \mathrm{nSv} / \mathrm{h}- \\
1 \mathrm{mSv} / \mathrm{h}\end{array}$ & $\begin{array}{l}10 \mathrm{nSv} / \mathrm{h}- \\
1 \mathrm{mSv} / \mathrm{h}\end{array}$ & $\begin{array}{l}10 \mathrm{nSv} / \mathrm{h}- \\
10 \mathrm{~Sv} / \mathrm{h}\end{array}$ \\
\hline $\begin{array}{l}\text { Gamma } \\
\text { Energy } \\
\text { Range }\end{array}$ & $>30 \mathrm{keV}$ & $\begin{array}{l}50 \mathrm{keV}- \\
1500 \mathrm{keV}\end{array}$ & $\begin{array}{l}40 \mathrm{keV}- \\
3000 \mathrm{keV}\end{array}$ \\
\hline Interfaces & USB & RS-485 & $\begin{array}{l}\text { USB, } \\
\text { RS-232, } \\
\text { RS-485 }\end{array}$ \\
\hline Made in & Germany & Poland & Czech Rep. \\
\hline
\end{tabular}

and unpredicted damages which can happen in the MPD detectors thus avoiding serious disaster.

The prototype dosimetry system is based on three types of probes equipped with RS-485 and/or USB communication interface. The first EKO-C is a simple mobile monitor of radioactive contamination produced by polish manufacturer POLON-EKOLAB [4] (Fig. 2). It is a pancake style mica window Geiger-Müller counter which has only one measuring tube inside. It has a rather small measuring range (up to $1 \mathrm{mSv} / \mathrm{h}$ ). The measurement error for $\mathrm{X}$ and gamma radiation does not exceed $\pm 15 \%$ (with reference to Cs-137). It is equipped with a half-duplex RS-485 connector. The second EGM-104 (Nuvia - Czech Rep.) is a more complex probe which consists of three GeigerMüller tubes (LND 7807 + LND 71210 + LND 71632) used for lower and higher dose rate range. The switching between tubes is performed according to the internal probe software. The probe can measure the ambient dose equivalent rate $\mathrm{H}^{*}(10)$ from $10 \mathrm{nSv} / \mathrm{h}$ up to $10 \mathrm{~Sv} / \mathrm{h}$ in total (Fig. 2) [5]. The measurement error for $X$ and gamma radiation is the same as for EKO-C dosimeter. It is furnished with WEIPU SF1213/P9 connector which has RS-232, RS-485 and USB interfaces inside. The third type of detectors is the Gamma-Scout (GS) [6] (Fig. 2) it is a Geiger counter that measures alpha, beta, gamma and $\mathrm{X}$-ray radiation like the EKO-C. Connection with this system is based on USB. It will be independent to RS-485 bus with changing up the reliability of the Dosimetry System. It consists of only one measuring tube inside like EKO-C. GS displays the results in $\mu \mathrm{Sv} / \mathrm{h}$ exactly. However, it should be remembered that its calibration was based on sources Cs-137 and similar Co-60 If the user works with other isotopes than Cs-137, he gets a reasonable dose rate, but not the precise dose rate. The Gamma Scout has a patented power flow to enable many years of battery driven operation without leakage of the battery. In case of lost of power GS will be working longer than the EKO-C detectors on their batteries. The EKO-C and GS will be located inside and outside of some racks with electronics as basic radiation level monitoring devices. The EGM-104 probes, which will act as a reference meter, are to be placed in a number of one on each floor of the platform with electronics (Fig. 1). A special dedicated cable was used to RS-485 communication. It consists of three pairs of twisted copper wires with

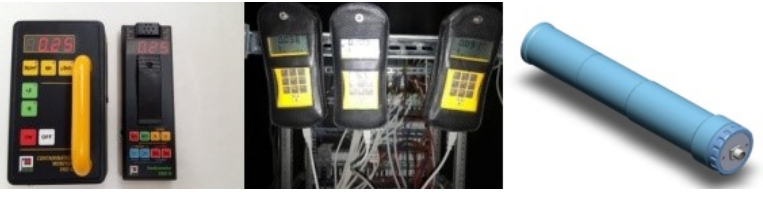

Figure 2. Left: EKO-C and EKO-D dosimetry probe. [6]. Center: Gamma-Scout dosimetry probe. Right: UM-EGM wide range gamma probe. $[7,8]$

a $0.22 \mathrm{~mm}^{2}$ cross section. It has a screen consisting of copper wires, the material of external insulation is PVC in purple color, and the diameter of the cable is $7.2 \mathrm{~mm}$. The cable is resistant to moisture, it can be used in the temperature range from $-40 \circ \mathrm{C}$ to $80 \circ \mathrm{C}$ at $250 \mathrm{~V}$ rated voltage. An important feature of the cable is its self-extinguishing in case of fire. All EKO-C and EGM probes are connected together and they constitute of the RS-485 bus along with the National Instrument's cRIO-9065 with the 4-port RS485 interface C module NI-9871 installed inside [7] (Fig. 3). The c-RIO has the built-in National Instrument Linux Real-Time Operating System and is equipped with powerful Field Programmable Array chip. The Compact RIO is the master and the probes are the slaves. The EKO-C dosimeters can only work with 9600 bit/s of the RS-485 interface rate. The EGM-104 probes can work with 9600 , 19200, 38400, 57600 and 115200 communication speed but the whole RS-485 bus ratio is determined by the slowest device so it is 9600 . The bus parameters are 8 data bits, 1 stop bit, no parity and no handshake.
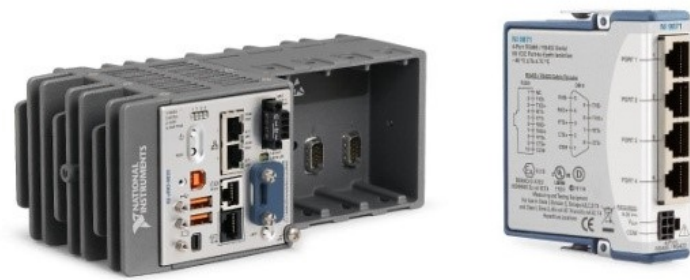

Figure 3. cRIO controller and NI-9871, RS-485 module [7].

We are going to use the National Instrument's LabVIEW [8] suit as a control and data visualizing software. The LabVIEW suit is installed on a PC computer to be equipped with an Ethernet card. It has to realize the following tasks:

- send control commands to each probe,

- receive data from probes,

- analyze the data and display it on the screen

- send alarms and messages to MPD supervisor and indicated persons (mobile application and/or e-mail),

- archive the data.

The CompactRIO unit will be connected to the USB port of the PC computer and it will collect all the read data from the probes. The software installed on the PC will 


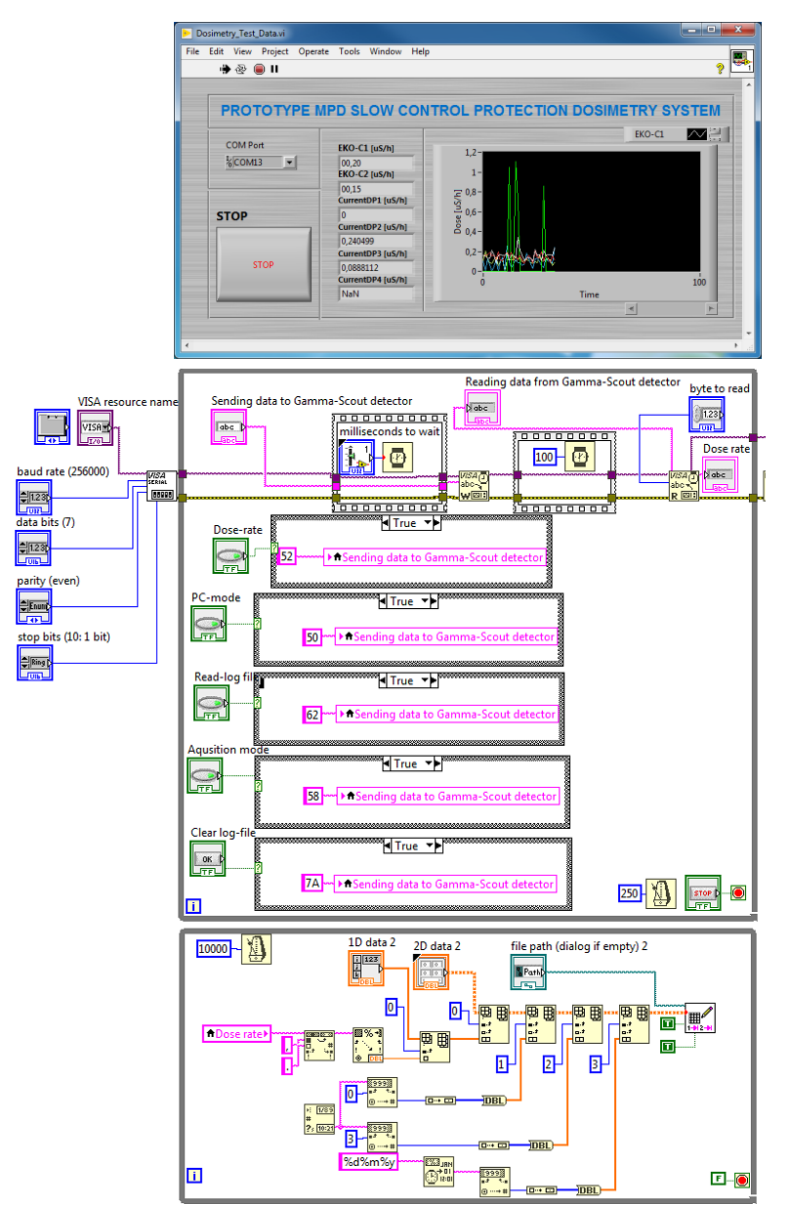

Figure 4. Up: Example screen from LabView control program. Bottom: Part of the LabView program block scheme for the Gamma Scout detectors.

be written in graphical LabVIEW language (Fig. 4). The software will be responsible for setting the probe parameters as well as collection of the data, presenting them in the visual form (diagrams, charts, etc.) and analyzing them which can finally result in the proper alarm decisions. All the collected data should also be archived in the Equipment database data Model (EqDb) [9]. The schematic diagram of the RS-485 bus prototype system is presented on Fig. 5. The access to the software is granted after the proper logging. There are three user access levels each one giving the different software possibilities. The first is the Guest level. This allows a user only to review the data and he has no privileges to change anything. The second level is called Technician. Such user except reviewing the data can also make changes in the probe settings but in the limited range. The last one is Administrator. In that case, a logged user can view all data and make unlimited changes in the dosimetry system settings, as well as full testing of the measurement system. This mode will be available only to selected people who know all the parameters of these devices. Next step is to choose the right alarming level in case of electronic equipment protection. It should be low enough to allow switching the MPD detector off before radiation damage of the Slow Control System occurs. The National Semiconductor Corporation

\section{FLOOR n}

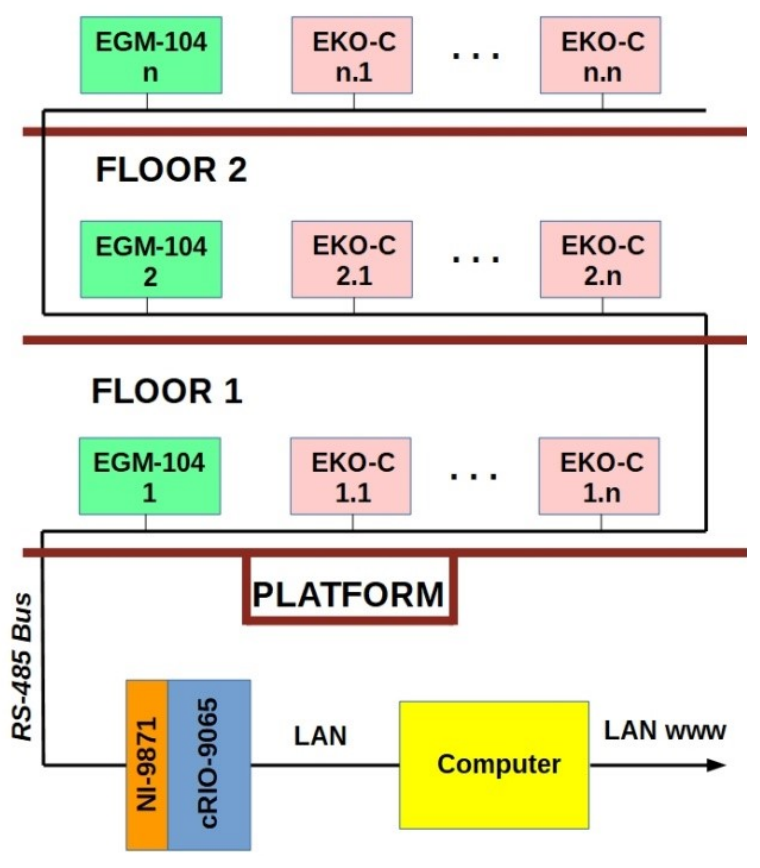

Figure 5. The schematic of the prototype dosimetry system for informing the MPD Slow Control system about unexpected irradiation.

(USA) [10] made the investigation of total ionizing radiation doses for different kinds of electronic devices. The most sensitive are CMOS devices, especially operational amplifiers, comparators, reference and data acquisition devices. The perimeter dose for them is about $100 \mathrm{~Gy}$. In case of people protection and according to ICRP 103 recommendations [11], reference exposure levels for workers and the public from single dominant sources for all exposures situation that can be controlled are as follow: (1) $1 \mathrm{mSv} /$ year or less, (2) greater than 1 to $20 \mathrm{mSv} /$ year, (3) greater than 20 to $100 \mathrm{mSv} /$ year. First band is for population, 2nd band for workers, 3rd band for workers in emergency cases.

\section{Summary}

The measuring system based on three different types of meters ensures greater accuracy and reliability of the entire measurement system. In the future, it is planned to extend the system with gauges capable of detecting pulse radiation (the current system is not very sensitive to this type of radiation), neutron radiation (the current system does not detect it) and a placement of dosimetry system for personnel performing service work on the platform where the Slow Control system is planned.

\section{Acknowledgements}

This work is partially supported by Research Program for the research group at JINR and research centres in Poland 
2019. This work is a part of Polish Consortium for NICA program (NICA-PL).

\section{References}

[1] Ref.: http://nica.jinr.ru/complex.php

[2] V. Golovatyuk, V. Kekelidze, V. Kolesnikov, O. Rogachevsky and A. Sorin, The Multi-Purpose Detector (MPD) of the collider experiment, Eur. Phys. J. A (2016) 52: 212

[3] K. Roslon, D. Dabrowski, M. Peryt, Design Brief of the Slow Control System for the MPD-TOF Detector, Act.Phys.Pol.B Vol.11 No.4 p.707 (2018)

[4] Technical documentation: Ref. http://www.polonekolab.com.pl/ekoc/ekoc.htm
[5] Technical documentation: Ref. http://nuviagroup.com/en/blog/product/components/nudetdetectors-and-probes/nudet-egm/

[6] Technical documentation: www.gamma-scout.com

[7] Technical documentation: Ref. http://www.ni.com/enus/shop/select/compactrio-controller

[8] Technical documentation: Ref. http://www.ni.com/enus/shop/labview.html

[9] M. Peryt, T. Traczyk, Equipment database data model, Act.Phys.Pol.B Vol.11 No.4 p.685 (2018)

[10] Radiation Owner's Manual, National Semiconductor Corporation, 1999.

[11] The 2007 Recommendations of the International Commission on Radiological Protection (ICRP) 